\title{
MULTIFIBRATIONS. A CLASS OF SHAPE FIBRATIONS WITH THE PATH LIFTING PROPERTY
}

\author{
Antonio Giraldo and Jose M. R. Sanjurjo, Madrid
}

(Received February 26, 1997)

\begin{abstract}
In this paper we introduce a class of maps possessing a multivalued homotopy lifting property with respect to every topological space. We call these maps multifibrations and they represent a formally stronger concept than that of shape fibration. Multifibrations have the interesting property of being characterized in a completely intrinsic way by a path lifting property involving only the total and the base space of the fibration. We also show that multifibrations (and also, with some restrictions, shape fibrations) have a lifting property for homotopies of fine multivalued maps. This implies, when the spaces considered are metric compacta, that the possibility of lifting a fine multivalued map is a property of the corresponding strong shape morphism and not of the particular map considered.
\end{abstract}

Keywords: shape fibration, multivalued map, path lifting property, strong shape

MSC 2000: 55P55, 55R05, 54C56

\section{INTRODUCTION}

A number of papers dealing with internal descriptions of the shape category have recently been published. The problem of giving internal characterizations of shape or of the most important invariants related to shape was already raised by K. Borsuk in his foundational papers of the theory (see, for instance, [B1, p. 146]). The problem has been attacked following two different lines. The first of them tried to give a noncontinuous description of the shape category while the second aimed at a description based on the use of multivalued maps. The first line is represented by the papers [F, S1, K, Č2] and the second by the papers [S2, S3, S4, GS, MoR, Č1]. The first connection between noncontinuity and shape morphisms was established

The authors are supported by DGES. 
by Felt in $[\mathrm{F}]$. In [S1] and [S3] respectively the first noncontinuous and multivalued characterizations of the shape category of compacta appear. The paracompact case was studied in $[\mathrm{K}]$ and $[\mathrm{MoR}]$. The characterizations for the class of all topological

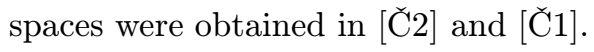

An important topic in shape is the theory of shape fibrations, first introduced by Mardešić and Rushing in [MR1] and [MR2] with some partial motivation from the previous work of Coram and Duvall $[\mathrm{CD}]$. In a recent paper $[\mathrm{G}]$, one of the authors has made a study of the theory of shape fibrations from the point of view of multivalued maps, proving that, in certain cases, the existence of liftings of strong shape morphisms can be reduced to an algebraic problem formulated in terms of the corresponding shape groups.

In the present paper we prove that a class of shape fibrations, which we call multifibrations, is characterized by a path-lifting property. This property involves only the total and the base space of the fibration and constructions based on them, hence giving an intrinsic characterization of this class of fibrations. It is an open problem whether the multifibrations coincide with the whole class of all shape fibrations.

We also present in the paper a lifting property enjoyed by the multifibrations (and, with some restrictions, by all shape fibrations) for homotopies of fine multivalued maps. When the spaces considered are metric compacta, then the homotopy class of a fine multivalued map is a strong shape morphism (see [GS]). Hence our result states that the possibility of lifting a fine multivalued map is a property of the corresponding strong shape morphism and not of the particular map considered.

For general information on shape theory we recommend the books by K. Borsuk [B2], J. M. Cordier and T. Porter [CP], J. Dydak and J. Segal [DS1], and S. Mardešić and J.Segal [MS]. We also recommend the collection of open problems [DS2] by Dydak and Segal. For a survey on shape fibrations see S. Mardešić $[M]$ and for relations between shape fibrations and strong shape see F. W. Cathey $[\mathrm{C}]$.

\section{Multifibrations}

Let $X$ and $Y$ be metric spaces. An upper semicontinuous multivalued function $F: X \rightarrow Y$ is a correspondence such that, for every $x \in X, F(x) \neq \emptyset$ is a closed subset of $Y$ and for every neighbourhood $V$ of $F(x)$ in $Y$ there is a neighbourhood $U$ of $x$ such that $F(U)=\bigcup_{y \in U} F(y)$ is contained in $V$. In the sequel, a map will be a continuous single-valued function, while upper semicontinuous multivalued functions will be called multivalued maps for short. $F$ is said to be $\varepsilon$-small if diameter $(F(x))<$ $\varepsilon$ for every $x \in X$. Two multivalued maps $F, G: X \rightarrow Y$ are $\varepsilon$-homotopic if there 
exists an $\varepsilon$-small multivalued map $H: X \times I \rightarrow Y$ such that $H(x, 0)=F(x)$ and $H(x, 1)=G(x)$ for every $x \in X$.

Let $E$ and $B$ be compact metric spaces and let $p: E \longrightarrow B$ be a map. We say that $p$ has the multivalued homotopy lifting property (MHLP) with respect to a topological space $X$ if for every $\varepsilon>0$ there exists $\delta>0$ with the following property:

Given $\delta$-small multivalued maps $F: X \longrightarrow E$ and $H: X \times I \longrightarrow B$ such that $d(p F(x), H(x, 0))<\delta$ for every $x \in X$, then there exists an $\varepsilon$-small multivalued map $H^{\prime}: X \times I \longrightarrow E$ such that $d\left(H^{\prime}(x, 0), F(x)\right)<\varepsilon, d\left(p H^{\prime}(x, t), H(x, t)\right)<\varepsilon$ for every $(x, t) \in X \times I$.

It was proved in $[G]$ that shape fibrations between compact metric spaces coincide with maps having the property MHLP with respect to any metric space $X$. A characterization in the same spirit has been given by Z. Cerin [Č3] in terms of relations.

Definition 1. We say that a map $p: E \longrightarrow B$ between compact metric spaces is a multifibration if it has the property MHLP with respect to any topological space $X$, in such a way that $\delta$ depends only on $\varepsilon$ and is, hence, independent on $X$.

The usual examples of shape fibrations (see [MR1]) are multifibrations. At the present moment we do not know if it is sufficient for $p$ to have the property MHLP with respect to all metric spaces in order to be a multifibration. Therefore the following is an open problem.

Problem. Is every shape fibration a multifibration?

Theorem 1 in $[G]$ guarantees, on the other hand, that every multifibration is a shape fibration. The following theorem states that multifibrations have characteristic path lifting properties. In order to formulate it, we must first introduce some terminology.

Given a compact metric space $E$ and given $\delta>0$ consider the set $P_{\delta}(E)$ of the $\delta$-small (that is, with diameter less than $\delta$ ) non-empty compact subsets of $E$ with the topology generated by the neighbourhood system

$$
\left\{B_{\varepsilon}(K) \mid K \in P_{\delta}(E), \varepsilon>0\right\}
$$

where $K^{\prime} \in B_{\varepsilon}(K)$ if $K^{\prime} \subset B_{\varepsilon}(K)$ in the usual sense. This topology is a restriction of the upper semi-finite topology studied by E. Michael [Mi] (see also Kuratowski $[\mathrm{Ku}]$ p. 175).

On the other hand, given a compact metric space $B$ and given $\delta>0$ let us consider the set $B_{\delta}^{I}$ of the $\delta$-small multivalued maps $\omega: I \longrightarrow B$ (or equivalently, the singlevalued maps from $I$ to $P_{\delta}(B)$ ), with the compact-open topology. This topology is 
equivalent to that generated by the neighbourhoods system $\left\{B_{\varepsilon}(\omega) \mid \omega \in B_{\delta}^{I}, \varepsilon>0\right\}$ where $\omega^{\prime} \in B_{\varepsilon}(\omega)$ if for every $t \in I$ there exists $t^{\prime} \in I$ with $d\left(t, t^{\prime}\right)<\varepsilon$ such that $\omega^{\prime}(t) \subset B_{\varepsilon}\left(\omega\left(t^{\prime}\right)\right)$.

Finally, given a surjective map $p: E \longrightarrow B$ and given $\delta>0$, we can define the set

$$
\Omega_{\delta}=\left\{(K, \omega) \in P_{\delta}(E) \times B_{\delta}^{I} \mid d(\omega(0), p(K))<\delta\right\} \subset P_{\delta}(E) \times B_{\delta}^{I},
$$

with the topology induced by the product topology of $P_{\delta}(E) \times B_{\delta}^{I}$.

The following result characterizes multifibrations in intrinsic terms, making no references to external spaces $X$ with respect to which to lift homotopies. This characterization reflects the path lifting properties of approximate nature for multifibrations.

Theorem 1. Let $E$ and $B$ be compact metric spaces and let $p: E \longrightarrow B$ be a surjective map. The following are equivalent:

(i) $p$ is a multifibration.

(ii) For every $\varepsilon>0$ there exist $\delta>0$ and a (single-valued) map $\lambda: \Omega_{\delta} \longrightarrow E_{\varepsilon}^{I}$ such that $d(\lambda(K, \omega)(0), K)<\varepsilon$ and $d(p(\lambda(K, \omega)(t)), \omega(t))<\varepsilon$ for every $(K, \omega) \in \Omega_{\delta}$ and every $t \in I$.

Pro of. We first see that i) implies ii). Let $\varepsilon>0$. Then there exists $\delta>0$ such that if $X$ is a topological space and $F: X \longrightarrow E$ and $H: X \times I \longrightarrow B$ are $\delta$-small multivalued maps such that $d(p F(x), H(x, 0))<\delta$ for every $x \in X$, there exists an $\varepsilon$-small multivalued map $H^{\prime}: X \times I \longrightarrow E$ such that $d\left(H^{\prime}(x, 0), F(x)\right)<\varepsilon$ and $d\left(p H^{\prime}(x, t), H(x, t)\right)<\varepsilon$ for every $(x, t) \in X \times[0,1]$.

Consider $X=\Omega_{\delta}, F: \Omega_{\delta} \longrightarrow E$ given by $F(K, \omega)=K$ and $H: \Omega_{\delta} \times I \longrightarrow B$ given by $H((K, \omega), t)=\omega(t)$. Then $F$ and $H$ are $\delta$-small multivalued maps such that

$$
d(p F(K, \omega), H((K, \omega), 0))=d(p(K), \omega(0))<\delta
$$

for every $(K, \omega) \in \Omega_{\delta}$. Hence there exists an $\varepsilon$-small multivalued map $H^{\prime}: \Omega_{\delta} \times I \longrightarrow$ $E$ such that for every $(K, \omega) \in \Omega_{\delta}$ and every $t \in I$ we have

$$
d\left(H^{\prime}((K, \omega), 0), F(K, \omega)\right)=d\left(H^{\prime}((K, \omega), 0), K\right)<\varepsilon
$$

and

$$
d\left(p H^{\prime}((K, \omega), t), H((K, \omega), t)\right)=d\left(p H^{\prime}((K, \omega), t), \omega(t)\right)<\varepsilon .
$$

Define $\lambda: \Omega_{\delta} \longrightarrow E_{\varepsilon}^{I}$ (single-valued) as $\lambda(K, \omega)(t)=H^{\prime}((K, \omega), t)$. Then $\lambda$ is well defined since for every $(K, \omega) \in \Omega_{\delta}$ we have that $\lambda(K, \omega): I \longrightarrow E$ is an $\varepsilon$-small 
multivalued map (since $H^{\prime}$ is). Moreover, $\lambda$ is continuous since given $\left(K_{0}, \omega_{0}\right) \in \Omega_{\delta}$ and $\eta>0$, then for every $t_{0} \in I$ there exists $0<\mu_{t_{0}}<\eta$ such that for every $K \in B_{\mu_{t_{0}}}\left(K_{0}\right)$, every $\omega \in B_{\mu_{t_{0}}}\left(\omega_{0}\right)$ and every $t \in I$ with $d\left(t_{0}, t\right)<\mu_{t_{0}}$ we have

$$
\lambda(K, \omega)(t)=H^{\prime}((K, \omega), t) \subset B_{\eta}\left(H^{\prime}\left(\left(K_{0}, \omega_{0}\right), t_{0}\right)\right)=B_{\eta}\left(\lambda\left(K_{0}, \omega_{0}\right)\left(t_{0}\right)\right) .
$$

Using the compactness of $I$ there exist $t_{1}, \ldots, t_{n} \in I$ such that

$$
I \subset\left(t_{1}-\mu_{t_{1}}, t_{1}+\mu_{t_{1}}\right) \cup \ldots \cup\left(t_{n}-\mu_{t_{n}}, t_{n}+\mu_{t_{n}}\right) .
$$

Consider $\mu=\min \left\{\mu_{t_{1}}, \ldots, \mu_{t_{n}}\right\}>0$. Then we have $\lambda(K, \omega) \in B_{\eta}\left(\lambda\left(K_{0}, \omega_{0}\right)\right)$ for every $K \in B_{\mu}\left(K_{0}\right)$ and for every $\omega \in B_{\mu}\left(\omega_{0}\right)$, since for every $t \in I$ there exists $t_{i} \in\left\{t_{1}, \ldots, t_{n}\right\} \subset I$ with $d\left(t, t_{i}\right)<\mu_{t_{i}}<\eta$ such that

$$
\lambda(K, \omega)(t)=H^{\prime}((K, \omega), t) \subset B_{\eta}\left(H^{\prime}\left(\left(K_{0}, \omega_{0}\right), t_{i}\right)\right)=B_{\eta}\left(\lambda\left(K_{0}, \omega_{0}\right)\left(t_{i}\right)\right) .
$$

Finally, $\lambda$ satisfies

$$
d(\lambda(K, \omega)(0), K)=d\left(H^{\prime}((K, \omega), 0), K\right)<\varepsilon
$$

and

$$
d(p(\lambda(K, \omega)(t)), \omega(t))=d\left(p\left(H^{\prime}((K, \omega), t)\right), \omega(t)\right)<\varepsilon
$$

for every $(K, \omega) \in \Omega_{\delta}$ and every $t \in I$.

We now prove that ii) implies i). Consider $\varepsilon>0$ and take $\delta>0$ and $\lambda: \Omega_{\delta} \longrightarrow E_{\varepsilon}^{I}$ according to the conditions of the theorem.

Let $X$ be a topological space and let $F: X \longrightarrow E$ and $H: X \times I \longrightarrow B$ be $\delta$-small multivalued maps such that $d(p F(x), H(x, 0))<\delta$ for every $x \in X$. We will see that there exists an $\varepsilon$-small multivalued map $H^{\prime}: X \times I \longrightarrow E$ such that $d\left(H^{\prime}(x, 0), F(x)\right)<\varepsilon$ and $d\left(p H^{\prime}(x, t), H(x, t)\right)<\varepsilon$ for every $(x, t) \in X \times[0,1]$.

Consider $G: X \longrightarrow B_{\delta}^{I}$ defined by $G(x)(t)=H(x, t)$. Then $G$ is a well defined map (this can be proved in a similar way as above for $\lambda$ ). Next define $G^{\prime}: X \longrightarrow \Omega_{\delta}$ by the expression $G^{\prime}(x)=(F(x), G(x))$. Then $G^{\prime}$ is a well defined map since

$$
d(p F(x), G(x)(0))=d(p F(x), H(x, 0))<\delta
$$

for every $x \in X$. Consider the composition

$$
X \stackrel{G^{\prime}}{\longrightarrow} \Omega_{\delta} \stackrel{\lambda}{\longrightarrow} E_{\varepsilon}^{I} .
$$

Then $\lambda G^{\prime}$ is a single-valued map. Finally, consider $H^{\prime}: X \times I \longrightarrow E$ given by $H^{\prime}(x, t)=\lambda G^{\prime}(x)(t)$. Then $H^{\prime}$ is a multivalued map since given $\left(x_{0}, t_{0}\right)$ and $\eta>0$, by 
the upper semicontinuity of $\lambda G^{\prime}\left(x_{0}\right)$ there exists $0<\eta_{1}<\eta$ such that if $d\left(t, t_{0}\right)<\eta_{1}$ then

$$
\lambda G^{\prime}\left(x_{0}\right)(t) \subset B_{\frac{\eta}{2}}\left(\lambda G^{\prime}\left(x_{0}\right)\left(t_{0}\right)\right) .
$$

Using the continuity of $\lambda G^{\prime}$ there exists a neighbourhood $U^{x_{0}}$ of $x_{0}$ in $X$ such that if $x \in U^{x_{0}}$ then

$$
\lambda G^{\prime}(x) \in B \frac{\eta_{1}}{2}\left(\lambda G^{\prime}\left(x_{0}\right)\right) .
$$

Then for every $t \in I$ there exists $t^{\prime} \in I$ with $d\left(t, t^{\prime}\right)<\frac{\eta_{1}}{2}$ such that

$$
\lambda G^{\prime}(x)(t) \subset B \frac{\eta_{1}}{2}\left(\lambda G^{\prime}\left(x_{0}\right)\left(t^{\prime}\right)\right)
$$

In particular, if $d\left(t, t_{0}\right)<\frac{\eta_{1}}{2}$ we have $d\left(t_{0}, t^{\prime}\right)<\eta_{1}$ and hence

$$
\lambda G^{\prime}\left(x_{0}\right)\left(t^{\prime}\right) \subset B_{\frac{\eta}{2}}\left(\lambda G^{\prime}\left(x_{0}\right)\left(t_{0}\right)\right) .
$$

Hence for every $(x, t) \in X \times I$ such that $x \in U^{x_{0}}$ and $d\left(t, t_{0}\right)<\frac{\eta_{1}}{2}$ we have

$$
H^{\prime}(x, t)=\lambda G^{\prime}(x)(t) \subset B_{\eta}\left(\lambda G^{\prime}\left(x_{0}\right)\left(t_{0}\right)\right)=B_{\eta}\left(H^{\prime}\left(x_{0}, t_{0}\right)\right) .
$$

Therefore $H^{\prime}$ is upper semicontinuous, and is $\varepsilon$-small since for every $(x, t) \in X \times I$ we have

$$
\operatorname{diam}\left(H^{\prime}(x, t)\right)=\operatorname{diam}\left(\lambda G^{\prime}(x)(t)\right)<\varepsilon .
$$

Finally, we have $d\left(H^{\prime}(x, 0), F(x)\right)=d(\lambda(F(x), G(x))(0), F(x))<\varepsilon$ and

$$
d\left(p H^{\prime}(x, t), H(x, t)\right)=d(p(\lambda(F(x), G(x))(t)), G(x)(t))<\varepsilon
$$

for every $(x, t) \in X \times I$.

\section{The Fine MUltivalued HOMOtOPY LIFTING PROPERTY}

Let $X$ be a topological space and let $Y$ be a metric space. A multivalued map $F: X \times \mathbb{R}_{+} \longrightarrow Y$ is called a fine multivalued map if it is upper semicontinuous and for every $\varepsilon>0$ there exists $r_{0} \in \mathbb{R}_{+}$such that $\operatorname{diam}(F(x, r))<\varepsilon$ for every $x \in X$ and every $r \geqslant r_{0} . F$ and $G$ are said to be asymptotic if for every $\varepsilon>0$ there exists $r_{0} \in \mathbb{R}_{+}$such that $d(F(x, r), G(x, r))<\varepsilon$ for every $x \in X$ and every $r \geqslant r_{0}$.

Two fine multivalued maps $F, G: X \times \mathbb{R}_{+} \longrightarrow Y$ are said to be homotopic if there exists a fine multivalued map $H: X \times[0,1] \times \mathbb{R}_{+} \longrightarrow Y$ such that $H(x, 0, t)=F(x, t)$ and $H(x, 1, t)=G(x, t)$ for every $(x, t) \in X \times \mathbb{R}_{+}$. 
Definition 2. Let $E$ and $B$ be compact metric spaces and let $p: E \longrightarrow B$ be a map. Let $X$ be a topological space. We say that $p$ has the fine multivalued homotopy lifting property (FMHLP) with respect to $X$ if given fine multivalued maps $F: X \times \mathbb{R}_{+} \longrightarrow E$ and $H: X \times I \times \mathbb{R}_{+} \longrightarrow B$ such that $p F$ and $H_{0}$ are asymptotic, there exists a fine multivalued map $H^{\prime}: X \times I \times \mathbb{R}_{+} \longrightarrow E$ such that $H_{0}^{\prime}$ and $F$, and $p H^{\prime}$ and $H$ are asymptotic.

In the following result we prove that multifibrations enjoy the property FMHLP with respect to every topological space $X$.

Theorem 2. Let $E$ and $B$ be compact metric spaces and let $p: E \longrightarrow B$ be a map. Let $X$ be a topological space and suppose that $p$ has the property MHLP with respect to $X \times I$. Then $p$ has the property FMHLP with respect to $X$.

As a consequence, if $p$ is a multifibration, then $p$ has the property FMHLP with respect to every topological space $X$. If $p$ is a shape fibration, then $p$ has the property FMHLP with respect to every metric space $X$.

Pro of. Suppose that $p$ has the property MHLP with respect to $X \times I$. Let $\left\{\varepsilon_{n}\right\}$ be a null sequence. Then there exists another null sequence $\left\{\eta_{n}\right\}$ such that, for every $n \in \mathbb{N}, \eta_{n} \leqslant \varepsilon_{n}$ and $\eta_{n}$ is associated to $\varepsilon_{n}$ by the property MHLP with respect to $X \times I$. On the other hand, if $p$ has the property MHLP with respect to $X \times I$, then $p$ has the property MHLP with respect to $X$. Therefore there exists a null sequence $\left\{\delta_{n}\right\}$ such that, for every $n \in \mathbb{N}, \delta_{n}<\frac{\eta_{n}}{3}$ and $\delta_{n}$ is associated to $\frac{\eta_{n}}{3}$ by the property MHLP with respect to $X$.

Let $F: X \times \mathbb{R}_{+} \longrightarrow E$ and $H: X \times I \times \mathbb{R}_{+} \longrightarrow B$ be fine multivalued maps such that $p F$ and $H_{0}$ are asymptotic. Then there exists an increasing sequence $\left\{k_{n}\right\} \subset \mathbb{N}$ such that

$$
\operatorname{diam}(F(x, r))<\delta_{n}, \quad \operatorname{diam}(H(x, t, r))<\delta_{n}, \quad d(p F(x, r), H(x, 0, r))<\delta_{n}
$$

for every $x \in X$, every $t \in I$ and every $r \geqslant k_{n}$. Then, for every $n \in \mathbb{N}$, there exists an $\frac{\eta_{n}}{3}$-small multivalued map $G_{n}: X \times I \longrightarrow E$ such that

$$
d\left(G_{n}(x, 0), F\left(x, k_{n}\right)\right)<\frac{\eta_{n}}{3}, \quad d\left(p G_{n}(x, t), H\left(x, t, k_{n}\right)\right)<\frac{\eta_{n}}{3}
$$

for every $x \in X$ and every $t \in I$. On the other hand, since $d\left(G_{n}(x, 0), F\left(x, k_{n}\right)\right)<$ $\frac{1}{3} \eta_{n}$ and $d\left(G_{n+1}(x, 0), F\left(x, k_{n+1}\right)\right)<\frac{1}{3} \eta_{n}$ we can consider an $\eta_{n}$-small multivalued 
$\operatorname{map} G_{n}^{\prime}: X \times\left(\{0\} \times\left[k_{n}, k_{n+1}\right] \cup I \times\left\{k_{n}, k_{n+1}\right\}\right) \longrightarrow E$ given by

$$
G_{n}^{\prime}(x, t, r)= \begin{cases}G_{n}(x, t) & \text { if } r=k_{n}, 0<t \leqslant 1 \\ G_{n}(x, 0) \cup F\left(x, k_{n}\right) & \text { if } r=k_{n}, t=0 \\ F(x, r) & \text { if } r \in\left(k_{n}, k_{n+1}\right), t=0 \\ G_{n+1}(x, 0) \cup F\left(x, k_{n+1}\right) & \text { if } r=k_{n+1}, t=0 \\ G_{n+1}(x, t) & \text { if } r=k_{n+1}, 0<t \leqslant 1 .\end{cases}
$$

Now, $G_{n}^{\prime}$ and $\left.H\right|_{X \times I \times\left[k_{n}, k_{n+1}\right]}$ are $\eta_{n}$-small multivalued maps such that

$$
d\left(p G_{n}^{\prime}(x, t, r), H(x, t, r)\right)<\eta_{n}
$$

for every $(x, t, r) \in X \times\left(\{0\} \times\left[k_{n}, k_{n+1}\right] \cup I \times\left\{k_{n}, k_{n+1}\right\}\right)$, and hence, applying the property MHLP there exists an $\varepsilon_{n}$-small multivalued map $G_{n}^{\prime \prime}: X \times I \times\left[k_{n}, k_{n+1}\right] \longrightarrow$ $E$ such that $d\left(G_{n}^{\prime}(x, t, r), G_{n}^{\prime \prime}(x, t, r)\right)<\varepsilon_{n}$ for every $(x, t, r) \in X \times\left(\{0\} \times\left[k_{n}, k_{n+1}\right] \cup\right.$ $\left.I \times\left\{k_{n}, k_{n+1}\right\}\right)$, and such that $d\left(p G_{n}^{\prime \prime}(x, t, r), H(x, t, r)\right)<\varepsilon_{n}$ for every $(x, t, r) \in$ $X \times I \times\left[k_{n}, k_{n+1}\right]$.

Finally, define $H^{\prime}: X \times I \times \mathbb{R}_{+} \longrightarrow E$ by

$$
H^{\prime}(x, t, r)= \begin{cases}G_{1}^{\prime \prime}\left(x, t, k_{1}\right) & \text { if } r \leqslant k_{1} \\ G_{n}^{\prime \prime}(x, t, r) \cup G_{n+1}^{\prime \prime}(x, t, r) & \text { if } r=k_{n+1}, n \in \mathbb{N} \\ G_{n}^{\prime \prime}(x, t, r) & \text { if } k_{n}<r<k_{n+1}, n \in \mathbb{N} .\end{cases}
$$

Then $H^{\prime}$ satisfies

$$
\operatorname{diam}\left(H^{\prime}(x, t, r)\right)<5 \varepsilon_{n}, \quad d\left(H^{\prime}(x, 0, r), F(x, r)\right)<2 \varepsilon_{n}
$$

and

$$
d\left(p H^{\prime}(x, t, r), H(x, t, r)\right)<\varepsilon_{n}
$$

for every $x \in X$, every $t \in I$ and every $r \geqslant k_{n}$.

The last statement is a consequence of the fact, proved in $[\mathrm{G}]$, that shape fibrations have the property MHLP with respect to every metric space.

It was proved in [GS] that if $X$ and $Y$ are compact metric spaces, then the strong shape morphisms from $X$ to $Y$ are just the homotopy classes of fine multivalued maps $G: X \times \mathbb{R}_{+} \longrightarrow Y$. Then, as a simple consequence of Theorem 2, we obtain the following result.

Corollary 1. Let $p: E \longrightarrow B$ be a shape fibration and let $X$ be a compact metric space. Suppose that $G, G^{\prime}: X \times \mathbb{R}_{+} \longrightarrow B$ are homotopic fine multivalued maps. 
Then $G$ can be lifted to $E$-in the sense that there exists a fine multivalued map $F: X \times \mathbb{R}_{+} \longrightarrow E$ such that $p F$ and $G$ are asymptotic - if and only if $G^{\prime}$ can be lifted to $E$. Hence, the fact that a fine multivalued map $G: X \times \mathbb{R}_{+} \longrightarrow B$ can be lifted to $E$ is a property of the strong shape morphism corresponding to $G$.

\section{References}

[B1] K. Borsuk: On movable compacta. Fund. Math. 66 (1969), 137-146.

[B2] K. Borsuk: Theory of Shape (Monografie Matematyczne 59). Polish Scientific Publishers, Warszawa, 1975.

[C] F. W. Cathey: Shape fibrations and strong shape theory. Topology Appl. 14 (1982), $13-30$.

[Č1] Z. Cerin: Shape theory intrinsically. Publ. Mat. 37 (1993), 317-334.

[Č2] Z. Čerin: Proximate topology and shape theory. Proc. Roy. Soc. Edinburgh 125 (1995), 595-615.

[Č3] Z. Čerin: Approximate fibrations. To appear.

[CD] D. Coram and P. F. Duvall, Jr.: Approximate fibrations. Rocky Mountain J. Math. 7 (1977), 275-288.

[CP] J. M. Cordier and T. Porter: Shape Theory. Categorical Methods of Approximation (Ellis Horwood Series: Mathematics and its Applications). Ellis Horwood Ltd., Chichester, 1989.

[DS1] J. Dydak and J. Segal: Shape Theory: An Introduction (Lecture Notes in Math. 688). Springer-Verlag, Berlin, 1978.

[DS2] J. Dydak and J. Segal: A list of open problems in shape theory. J. Van Mill and G. M. Reed: Open problems in Topology. North Holland, Amsterdam, 1990, pp. $457-467$.

[F] J. E. Felt: $\varepsilon$-continuity and shape. Proc. Amer. Math. Soc. 46 (1974), 426-430.

[G] A. Giraldo: Shape fibrations, multivalued maps and shape groups. Canad. J. Math 50 (1998), 342-355.

[GS] A. Giraldo and J. M. R. Sanjurjo: Strong multihomotopy and Steenrod loop spaces. J. Math. Soc. Japan. 47 (1995), 475-489.

[K] R. W. Kieboom: An intrinsic characterization of the shape of paracompacta by means of non-continuous single-valued maps. Bull. Belg. Math. Soc. 1 (1994), 701-711.

[Ku] K. Kuratowski: Topology I. Academic Press, New York, 1966.

[M] S. Mardešić: Approximate fibrations and shape fibrations. Proc. of the International Conference on Geometric Topology. PWN, Polish Scientific Publishers, 1980, pp. 313-322.

[MR1] S. Mardešić and T. B. Rushing: Shape fibrations. General Topol. Appl. 9 (1978), 193-215.

[MR2] S. Mardešić and T. B. Rushing: Shape fibrations II. Rocky Mountain J. Math. 9 (1979), 283-298.

[MS] S. Mardešić and J. Segal: Shape Theory. North Holland, Amsterdam, 1982.

[Mi] E. Michael: Topologies on spaces of subsets. Trans. Amer. Math. Soc. 71 (1951), $152-182$.

[MoR] M. A. Morón and F. R. Ruiz del Portal: Multivalued maps and shape for paracompacta. Math. Japon. 39 (1994), 489-500.

[S1] J. M. R. Sanjurjo: A non-continuous description of the shape category of compacta. Quart. J. Math. Oxford (2) 40 (1989), 351-359. 
[S2] J. M. R. Sanjurjo: Multihomotopy sets and transformations induced by shape. Quart. J. Math. Oxford (2) 42 (1991), 489-499.

[S3] J. M. R. Sanjurjo: An intrinsic description of shape. Trans. Amer. Math. Soc. 329 (1992), 625-636.

[S4] J. M.R. Sanjurjo: Multihomotopy, Cech spaces of loops and shape groups. Proc. London Math. Soc. (3) 69 (1994), 330-344.

Authors' addresses: Departamento de Matemática Aplicada, Facultad de Informática, Universidad Politécnica, Campus de Montegancedo, Boadilla del Monte, 28660 Madrid, Spain, e-mail: agiraldo@fi.upm.es; Departamento de Geometría y Topología, Facultad de Matemáticas, Universidad Complutense, 28040 Madrid, Spain, e-mail: jose_sanjurjo@mat.ucm.es. 